\title{
Semantic Management of Urban Traffic Congestion
}

\author{
Arshad Shakil \\ University of South East Norway, Kongsberg \\ 888286@usn.no
}

\author{
Radmila Juric \\ University of South East Norway, Kongsberg \\ rju@usn.no
}

\begin{abstract}
Urban traffic congestion is a problem which affects the world and is related to the massive urbanization and excessive number of cars on our streets. This causes a variety of problems, from economical/financial and health-related, to environmental warnings caused by high $\mathrm{CO}_{2}$ and $\mathrm{NO}_{2}$ emissions. This paper proposes a novel software engineering solution, which generates a software application aimed at individual drivers on urban roads, in order to help and ease overall congestion. The novelty is twofold. We target individual drivers in order to motivate them to re-think the purpose and goals of each journey they take. Consequently, the proposed software application enables reasoning upon various options an individual driver may have and helps in choosing the best possible solution for an individual. Our software application utilizes reasoning with SWRL enabled OWL ontologies, which can be hosted by any software application we run in our cars, ready to assist in driving, and implemented in Android / iOS environments.
\end{abstract}

\section{Introduction}

For the past 70 years, the world has experienced high levels of urbanization [1]. According to the United Nations, the urban population of the world has grown from approximately 750 million in 1950 to about 4.2 billion in 2018. By 2050, about $68 \%$ of the world's population will live in urban areas[2]. The urban agglomeration has led to an increase in wealth but also to the rise in consumption and pollution. With an increase in population, the need for better and bigger urban infrastructure is obvious and thus making it expensive for the cities to sustain the growth of population and vehicles on their roads [3] [4]. It would be interesting to see if there is any correlation between the speed of urbanization and economic growth at a global level, but the link is not strong. When looking at the increase in urbanization levels, with China going from $10-20 \%$ to $50-60 \%$ by 2011 , most countries in the world show economic growth. However, some countries show zero or negative growth.
Therefore, urbanization is a complex issue and has a complicated relationship with the world economies.

The number of cars in the world has been increasing since 1950 and doubling every ten years [5]. In 1985 the world hit the 500 million mark, in 2010 the number was 1 billion, and by the end of 2015, there were 1.28 billion cars worldwide[6] The increase has been the biggest in Asia. In 2016, 70.5 million cars were manufactured, where one-third of all cars are produced in China [7]. The increase in car numbers also means that there are fewer people per car. In 2017 there were approximately 268 million registered vehicles in the US [8], including passenger cars, small/big cars, motorcycles, trucks, and buses privately owned. Considering that the population on the $1^{\text {st }}$ April 2017 was 324 million [9], it shows that the person-to-vehicle ratio at that time was 1.21.

Consequently, urban traffic congestion exists everywhere. One major factor causing congestion is the daily commute which causes peak rush-hours in the morning[10] and after work [11]. Incidents such as accidents, planned roadwork, parking on the roads, and breakdowns[12] can contribute further to congestion. The weather, poorly configured and timed traffic management systems and special events are also incidents that can increase the congestion [13]. However, there have been laws describing the nature of peak-hour congestion. In 1962 Anthony Downs proposed The fundamental law of congestion [14], which is the same as Parkinson's Second Law applied to traffic: On urban commuter expressways, peak-hour traffic congestion rises to meet maximum capacity. Cyril N. Parkinson's Second Law, used in economics, states that: Expenditure rises to meet income [15]. They both show that if there is capacity, it will be utilized to the maximum. If we build bigger roads, more people will flock to them and create congestion.

In 1992, Downs proposed another theory called The principle of triple convergence. This was an extension to the law he proposed 30 years earlier[16]. In both cases, he argued that peak-hour congestion is a result not only of lacking road infrastructure, but it has roots in economic, psychological, social, and location-based consideration. 
An interesting approach to address the psychology of drivers is illustrated through the application of game theory, where drivers, traffic lights, and all constituent parts of urban traffic are considered "players." In the Nash Equilibrium [17] it is assumed that each player knows other players' equilibrium strategies and no player can change his/her own strategy unilaterally with gains. Individuals can receive incremental benefit from changing actions. The set of strategy, choices and corresponding payoffs, constitute a Nash equilibrium in this game (traffic). Nash and Downs show how psychology and strategy play a role in the search for a potential solution for urban traffic congestion.

In this paper, we would like to look at this problem from a slightly different perspective. We would like to know if "individuals (individual drivers) are very much responsible for overall urban traffic congestion. Why do we run away, from the fact, that drivers in their cars may cause traffic congestion?

The idea of targeting individual drivers, to ease urban traffic congestion, is not new in our research. However, this paper proposes a generic software architectural model, which fits any situation in urban traffic and produces assistance to individual drivers. Talking about the needs of a driver, means motivating drivers to RE-THINK the purpose and goals of every journey he/she undertakes. Therefore, the novelty of this research is in the shift in thinking on how to address the urban traffic congestion: we should start from an individual driver. Apart from using all available software applications in their cars, drivers may integrate this proposal into the car's environment. The proposal will help with the reasoning upon semantics collected in traffic, and give the best possible answers to a driver in a particular situation. Drivers are being seen as responsible for finding themselves in traffic congestion and therefore the proposal helps in understanding: Why am in this congested road (again)? Could have I avoided this? How do I resolve this problem now?

The paper is organized as follows.

Section 2 summarize the problem by outlining current solutions to urban traffic management, which are related to urban traffic congestion. It is obvious that there are no universal solutions to the problem and research on resolving traffic congestion is scattered and fragmented. Section 3 looks at the examples from peer-reviewed papers, where Semantic Web Rule Language (SWRL) enabled Ontology Web Language (OWL) ontologies are used in urban traffic management and the deployment of OWL ontologies in particular. This would help to evaluate the proposal. Section 4 gives a Scenario of traffic congestion in the Oslo municipality, in order to illustrate both: conceptual model of the proposal and its implementation. Therefore, the Proposal section contains a generic and reusable software architecture for the proposal and conceptual OWL model with reasoning. The implementation section shows a prototype in the Protégé tool, in which OWL model is populated with the semantic defined in the Scenario. The reasoning is defined and performed in order to obtain the answer to questions the driver may ask in a particular situation in traffic. Conclusions debate results of this research.

\section{The Problem}

Problems caused by urban traffic congestion are very well known. Environmental issues are related to $\mathrm{CO}_{2}$ emissions, global warming and climate change. The emissions from vehicles and other forms of transport is a serious problem: the release of Sulphur Dioxide $\left(\mathrm{SO}_{2}\right)$ and Nitrogen Oxides $\left(\mathrm{NO}_{\mathrm{x}}\right)$ can lead to acid rain, harmful to ecosystems and can lead to the death of trees and fish [18]. High levels of Sulphuric Acid $\left(\mathrm{H}_{2} \mathrm{SO}_{4}\right)$ and $\mathrm{SO}_{2}$ were among the contributors to the catastrophe in London in the 50s [19]. This is not all. The time we spend in traffic is substantial and time is money! If we look at the cost of traffic congestion for each driver, wasting fuel and increased vehicle operating costs are significant [20]. According to INRIX[21], congestion cost for Americans were nearly $\$ 87$ billion in 2018, while in the U.K. the figure is close to $£ 8$ billion. Congestion increases risks of accidents, which in tur cause injuries or death $[22,23]$ However, the cost of a crash per person is a lot higher than the cost of congestion per person, regardless of the size of urban areas, but the cost of congestion is lower as the size of the metropolitan areas gets smaller. The loss of productivity is another major factor triggered by congestion[24]. More time spent in traffic means less time to get your work done. A report done by McKinsey has shown that cities can lose $2-4 \%$ of their GDP due to congestion[25].

Health-related problems caused by congestions range from accidents, injury and even death to ambient pollution which harms every single individual. Pollution causes respiratory problems, cancer [26] and has also been linked to childhood asthma [27], cardiovascular problems and stroke[28]. Pollution in the form of Particulate Matter (PM) is also claimed to be one of the causes of lower life expectancy[29] and premature death. PM is categorized by the size of the particle, where $\mathrm{PM}_{10}$ are all particles with a diameter of 10 micrometers or smaller. $\mathrm{PM}_{10} \mathrm{PM}_{5} \mathrm{PM}_{2.5} \mathrm{PM}_{1}$ are definitions that have been used, where $\mathrm{PM}_{10}$ and $\mathrm{PM}_{2.5}$ are the most commonly mentioned[30] when it comes to urban traffic emissions. A report done by the Energy policy institute at the University of Chicago has shown that air pollution can cut global life expectancy by 
nearly two years[31]. Another study has also shown the positive effect of decreasing pollution and the increased life expectancy[32].

We are all aware of the problems above. The increase in the numbers of cars internationally, with the increased urbanization, means that most of the cars are now located in large cities and there is a cap for the max number of vehicles which could travel on urban roads. The following questions are not new, but it is worthwhile repeating them:

- What have we done so far in this problem domain?

- How does the world address these quite scary facts?

- What are scientists supposed to do because, so far, we have not seen any successful and lasting solution for the problem?

Here is an overview of a selection of solutions found in peer-reviewed papers.

A congestion charge has been implemented in Stockholm[33] and London[34] with initially relatively good results, but it remains to be seen if their long-term impact will be positive. There are attempts to introduce tolls and fees, and differentiated road pricing schemes[35, 36], in order to see if they can help in minimizing impact traffic congestion has on health and environment [37] and on drivers behavior[38] .

There are solutions which use vehicular ad-hoc networks[39] where vehicles exchange traffic information[40] to create intelligent traffic systems[41] and traffic management systems[42, 43]. Another strategy is to use traffic signal controllers[44] at intersections to optimize the traffic flow, and introduce adaptive road routing [45] [46] [47]. Traffic congestion predictions are often performed with surveillance data [48] [49] [50] or data generated by vehicles [51], enhanced with Bluetooth technology [52].

At the time of writing, there were no published papers involving GPS backed navigation software. They are used in personal Apps and they route drivers to their destination, usually by showing the shortest path and travel time. They provide traffic decision support for an individual driver, but they do not take into consideration where other drivers are going and may contribute to increasing congestion [53] In traffic decision making, drivers usually select the shortest route because they see it as an optimal solution. However, the authors of [54] claim that, by choosing a less selfish route, drivers can improve traffic flow: total congestion can be reduced by up to $30 \%$. Therefore, congestion can be avoided by focusing on the psychological aspect of driving and introducing socially-aware traffic routing for individual drivers.

\section{Related Work}

This section overviews research which uses SWRL enabled OWL ontologies for addressing urban traffic congestion. There are mostly formal ontologies defined as controlled vocabularies and knowledge bases: they do not exploit reasoning with SWRL for guiding individual drivers in traffic. Therefore, it was very difficult to find peer-reviewed papers, which are closely related to this research.

In[55] the authors illustrate an Advanced Driver Assistance System (ADAS) that helps improve driving safety for electric cars in urban areas. The ontology models the environment within and outside a vehicle and defines various situation in traffics, which may warn or even alarm drivers on potential danger. In [56] an instance of ADAS is fed by data related to the current traffic situation, including sensor-generated data in the vehicle. Their ontology describes the situation in traffic at interactions and between different entities and helps in raising driver's context awareness. A similar approach was also proposed in [57]. Their ontology also models the traffic situations at different intersections, but adds data from traffic infrastructure, maps, traffic rules, and other sensor-generated data, and creates semantics for defining context in traffic.

$\operatorname{In}[58]$ the authors propose an ontology which helps drivers to plan a trip with public transport, which includes bus, metro, train, and tram. They take into consideration factors like price, day of the week, special events, the infrastructure of the public transportation and points of interest (ATM, restaurant, grocery store, etc.). They also offer to switch modes of transportation. The semantic stored in the ontology is strengthen by SWRL rules.

In [59] a user creates the content of OWL ontology, but a hierarchical analytic process (AHP), based on qualitative and/or quantitative criteria is used instead of reasoning. The model allows for personal preferences to create personalized advice to route planning.

In [60] a 3D simulator creates data for predicting traffic accidents. The tests they performed generate semantics for deriving SWRL rules, as a part of the prediction mechanism. In [61] the authors predict potential incidents, based on a traffic accident database, which is used for defining and building an ontology. However, its content is retrieved using SPARQL. Ontology in [62] models situations in traffic and SWRL is used for inferring traffic regulations, compliant with driving, in any country in the world.

In [63] a traffic jam control system, OnTraJaCS, reroutes drivers to ease congestion, at a system-wide level, even though some individual drivers may experience longer travel times than others. OWL is used for detecting congestion. 
In this decade we started associating situations in traffic with Internet-of-Everything and Internet-ofVehicles [64] [65], [66]. This will open door for new software solutions in which participants in traffic are able to share data and functionalities of software which surrounds us and they might address traffic congestion differently. The deployment of ontologies in such environment, and reasoning upon them, might be one of the solution for future traffic management, which would address traffic congestion with new computational models.

\section{The Scenario}

In this section we describe a scenario from a set of urban roads in the Oslo municipality. We use it for

- Defining a context in traffic which might be of interest to readers;

- Underpinning the proposed conceptual solution;

- Populating OWL ontologies (individuals) and define the object properties between them according to the semantics from the scenario.

Let us assume that Mr Lars, who lives in Drammen, commutes to Oslo every weekday. He drives from the city center in Drammen towards Lier and gets on the motorway (E18). He then follows E18 for $31 \mathrm{~km}$ before going onto Ring Road 3 (Rv 150). He then follows Ring Road 3 for approximately $7.7 \mathrm{~km}$ before parking near Ullevål football stadium. From there, he walks for 2 minutes to get to his office. This trip is $42 \mathrm{~km}$ long, and according to his GPS software application, it should take him $40 \mathrm{~min}$. to reach the destination. However, Lars knows that it takes him approximately 1 hour from his home before he walks into his office.

He is used to congestion on the motorway in the mornings, so he likes to leave home early just in case the traffic jam is particularly bad. He keeps thinking how he should use public transport and trains, but his drive to work gives him freedom and flexibility. When he sometimes decides to take public transport, he has to use the train/tram/bus, and software applications from Ruter and Vy to plan his trips and buy tickets in advance. Lars also works from home twice a week, but he does not use the possibility very much, because he likes his colleagues and would rather be at work.

While driving his car Lars is connected to his cellphone and then to any other network / car using Bluetooth. He then uses Google Maps navigation whenever necessary. His workplace, which is next to the Ullevål football stadium is the destination, entered into these Apps, and if he wants to be informed, about possible congestion and traffic incidents through the app, the messages come automatically. He also listens to the DAB radio, in case they broadcast traffic-related information.
One day Lars has a meeting at work and decides to leave home 1 hour and 20 minutes before the meeting. That is approximately 40 minutes longer than what Google Maps tells him, and 20 minutes more than his usual commute time. While driving on the E18, he learns about an accident on Ring Road 3, which is $2 \mathrm{~km}$ before his destination. The traffic is not moving, and the DAB radio recommends everyone to avoid Ring Road 3 going east from Asker.

He tries to use Google Maps navigation to find other routes, but the app suggests alternative routes for Ring Road 2 instead of 3 . He quickly sees that he will miss the meeting at work even if he takes the alternative suggested routes, because the new time to arrival, calculated by the app, shows that he will be late. It appears that all the traffic has been diverted and is going east towards Ring Road 2, thus flooding all side roads near the accident. Even if Lars takes a different route, he knows there will be congestion on any of these those roads, and he will be late anyway. Lars then starts wondering what he should do next to reach his destination. He remembers that he has the possibility of joining the meeting online, through Skype, but he would prefer to be there in person.

Lars was thinking about the train, or metro to get to work, and the following sources of traffic information: Google Maps navigation, Vy/Ruter apps can help him to plan his journey, but he is not sure what exactly would be the best option. His question is

"What is the smartest thing for me to do now, and is there any way for me to be there in person without being delayed?"

Google Maps does not have the answer he needs, he will be late anyway, and he is unsure what do next. He may have choices generated by a software application, according to the traffic context/situation. For example Lars could have been recommended the following:

1. Continue his regular route but get there too late

2. Continue his regular route but join Skype through $4 \mathrm{G}$ connection

3. Follow Google Maps re-route but get there too late

4. Abandon trip, go home and join late, via Skype

5. Abandon trip, go to a place with $\mathrm{WiFi}$ and join via Skype

6. Park the car, take the bus/train and maybe get there in time

None of the software applications available for Lars could recommend exactly what would be best for him. It's not always possible to get all the advice we need from Driver Assistance Systems (DAS) like Google Maps navigation. In Google Maps navigation, there is information regarding vehicular GPS navigation and you can use public transportation, but Google Maps does NOT give any other option, which could address 
personal needs of a driver and become specific to the context in the traffic.

Lars might benefit from the proposal given in this research: he will be recommended a suitable option, automatically generated by software at any moment in his journey to work.

\section{The Proposal}

\subsection{Software Architecture}

The proposed software architecture (SA) is in Figure 1. The SA is a layered and component-based software architectural style where computational components separate user interfaces from the persistence and data repositories. It follows the Model View Controller (MVC) pattern and allows numerous types of computations to share data repositories within one single software application.

The SA is deployed using Java technologies and thus uses servlet or enterprise java beans, which are all accessible through Integrated Development Environment, such as NetBeans. The SA is selfexplanatory: Java Servlets, Java classes, and User Interface (UI) are used according to the definition of the MVC pattern. There are two main servlets (computations), which belong to two different pathways of the SA model.

a) The first pathway on the left part of Figure is reserved for manipulating data which exists in the environment/context through the UI.Situaiton user interface. It contains data which describe the current situation (i.e. it identifies "context") on the road. Data is available from external sources, such as Google maps, traffic signs, road layouts, traffic news and bulletins and many more.

b) The second pathway, on the right side of the SA model in Figure 1, is reserved for ChooseRoute computations. This means that the application connects to our OWL model and performs reasoning with SWRL, to choose the best possible route for a driver in the identified "context" (from the first computational pathway).

Therefore the reader should perceive the abstractions from the SA model as a software application split into two parts. The left-hand side contains all possible repositories, which are defined in this Scenario, and which create a context. This, in turn is shared with the reasoning process on the right-hand side of the SA model.

The ontology on the right-hand side can be automatically populated with data from persistence from the left part of the SA. However, the driver can give his/her own information using its user interface
UI.choose route plus (i) specify his/her preferences, while being in the traffic, and (ii) add what the purpose of his trip is, as indicated in the Scenario.

This paper focuses on the right side of the SA in Figure 1 (the dotted, blue part). For readers who would be interested in the way the reasoning model, using SWRL enabled OWL ontologies, has been implemented through OWL-API, within a Java application, we suggest reading [67]. For readers interested in finding out how to populate OWL ontologies automatically from the existing persistence, reading [68] is recommended.

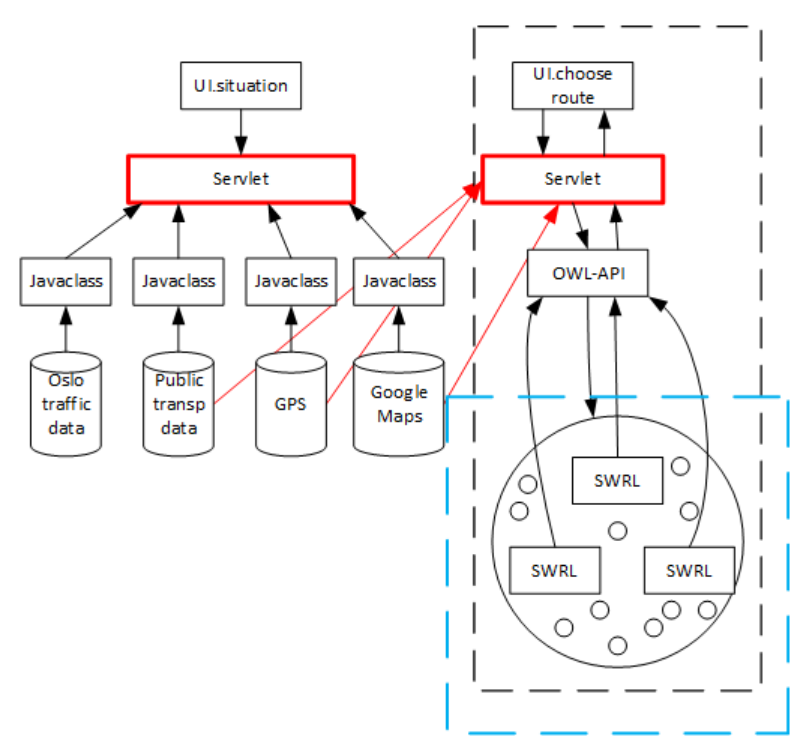

Figure 1: Software architecture of the proposal

These two pathways of computations from Figure 1 are explained further. The servlet Situation, is used to describe the context in which a driver happens to be. The context is generated from information available to the driver. The data is collected through Google Maps (map over roads, notifications about accidents, route suggestions), location data from GPS, persistent data from the Oslo municipality (road infrastructure, signs, speed limits, traffic lights, etc.), or data from apps regarding the public transportation (train/bus/metro departures, delays in public transportation, etc.).

Servlet ChooseRoute (above the right, blue dotted section of the conceptual model) would assume that relevant data about the context in traffic, has been inserted into OWL ontology and demonstrates how reasoning with SWRL create the best possible decision for an individual driver, and according to the "context" identified in the first computational pathway. However, in this part of the computational model, individual preferences of the driver and specificity of his/her journey, is entered though the UI.ChooseRoute 
and therefore the purpose of the journey, personal preferences, and options/routes the driver may have could have been entered into the reasoning systems either manually or automatically

\subsection{OWL Model and Reasoning}

Figure 2 shows a generic OWL model and the reasoning process of the proposal.

The model is layered and contains up to $\mathrm{n}$ layers in which we pair individuals of PREFERENCE and PURPOSE classes with either ROUTES or RESULT class.

ROUTES class contains individuals of all possible routes we may have, which were either generated earlier (through the first computational pathway from Figure 1) or entered through the application.

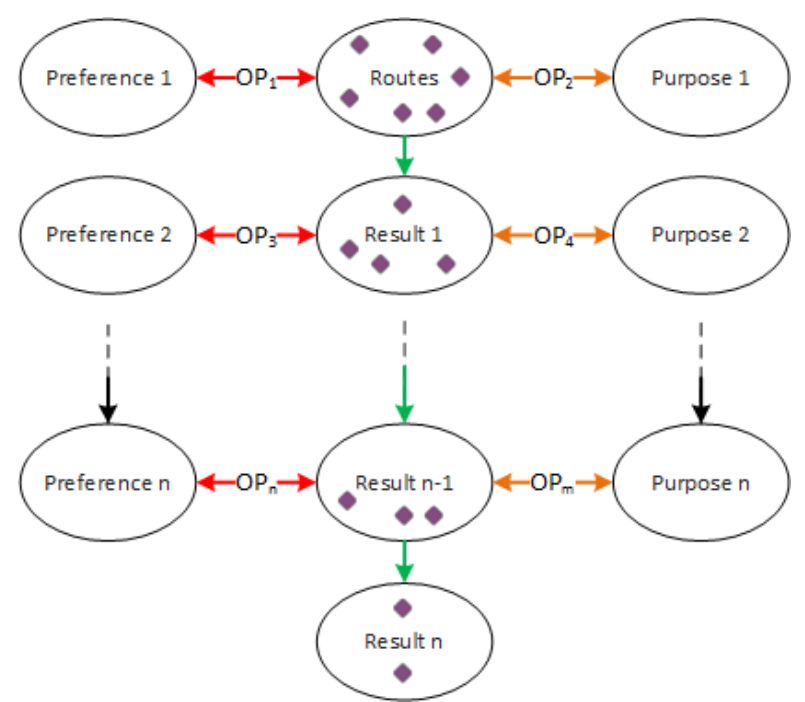

Figure 2: Conceptual OWL model and reasoning

PREFERENCE and PURPOSE classes contain individuals entered by a driver, which store exactly what the driver would want to have: which preferences he/she might have when being in the situation where he has to choose the best route. Individuals of PURPOSE class might not be as numerous as individuals of PREFERENCES class, but they will contain information on the purpose of each type of a journey the driver undertakes.

Object properties, marked with amber bidirectional arrow are defined as hasPreference and has_Purpose and they create semantic overlapping between individuals of (PREFERENCE and ROUTE/RESULT) and (PURPOSE and ROUTE/RESULT) classes.

The first SWRL rule (SWRL rules are denoted with green one-directional arrow) shows the inference and the way we move individuals of ROUTES class to
RESULT1 class. This means that the RESULT1 class will contain available routes, which satisfy criteria in the first SWRL rule.

For all other SWRL rules, we use object properties between (PREFERENCEk and RESULTj) and (PURPOSEk and RESULTj) classes. This means that individuals of PURPOSE class are travelling down the model into RESULT1, RESULT2, RESULTn classes (only if they satisfy object properties defined in their SWRL rules.

Obviously, there can be up to $\boldsymbol{n}$ different possibilities of reasoning (horizontal lines in Figure 2), which is determined by the number of object properties defined between (PREFERENCEk and RESULTj) and (PURPOSEk and RESULTj) classes.

In short, individuals from the ROUTES class are "filtered" by running SWRL, rules one after another, in a chain and according to pre-defined object properties which are used in SWRL rules. We are reasoning upon the result of previous reasoning. A similar way of chaining the execution of SWRL rules has been proposed in [69]. However, the proposed model has two sets of object properties per each filtering iteration, which uses object properties hasPurpose and hasPreference

\section{Implementation}

The illustration of the implementation of the conceptual model from Figure 2 is in Figure 3. The semantic from the scenario is used to determine the number of classes in the implementation model, their individuals and object properties.

In Figure 3 we specify up to $\boldsymbol{n}$ different classes for RESULT, PREFERENCES and PURPOSE, but in reality, we will use only three layers of the reasoning from the conceptual model. According to the Scenario, Mr Lars does not have more than 3 entries (individuals for PREFERENCES and PURPOSE classes). Therefore, the model form Figure 3 is self-explanatory. It is created in Protégé editing tool.

Table 1 shows important semantics: object properties defined between individuals of domain and range classes. The Purpose class is called Purp, and the Preference class is called Pref.

Due to space limitation, the exact semantic overlapping between the individuals has not been shown (only between the DOMAIN and RANGE classes). Also only individuals which are of interest to us in this particular context (Scenario) have been listed. We may have any number and type of individuals of RANGE classes: they are actually driver's entries! 


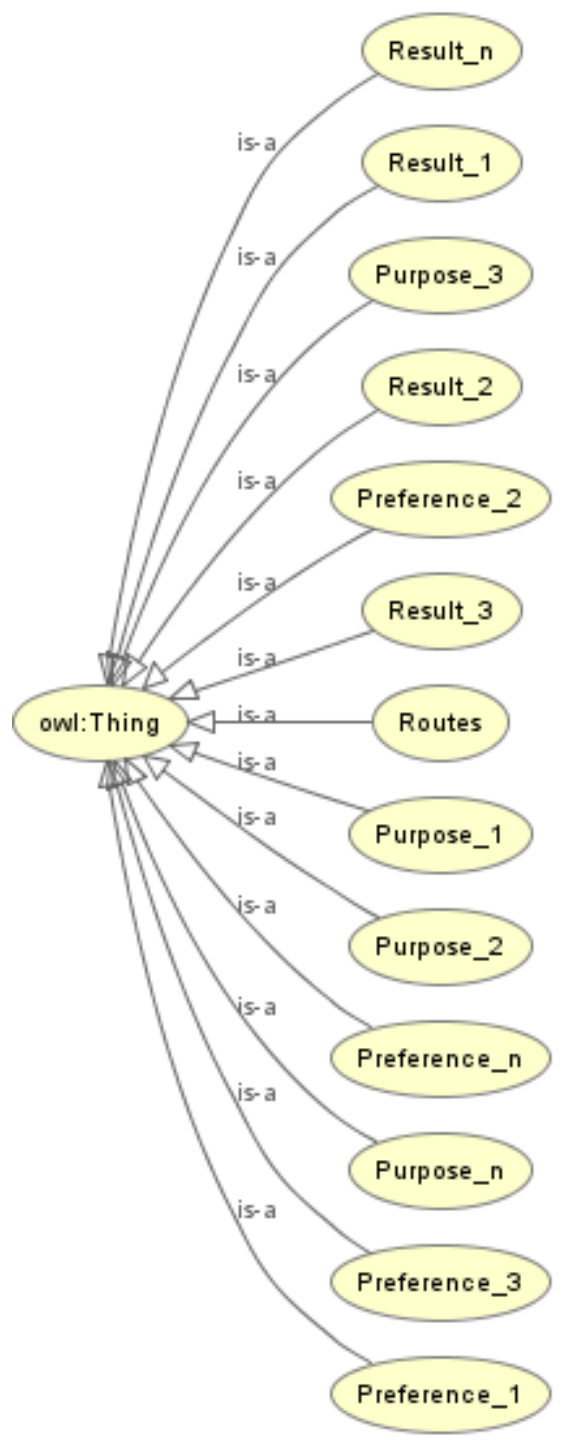

Figure 3: Ontological model for the scenario

Figures 4 is a Protégé screenshot of individuals of ROUTE class. They have been taken form the Scenario and entered into OWL ontology.

For the implementation of our solution, the SWRL rules defined in Figure 5 must be run. It shows three SWRL rules, which were run for each level of reasoning. The number of rules is dictated by the number of individuals in the PURPOSE and PREFERENCES classes, as explained in the conceptual model.

The rules in Figure 5 are generic, with no hard coding, and thus reusable for any number of individuals in the OWL ontology.

Figures 6-8 shows Protégé screen-shots of individuals of classes RESULT1, RESULT2 and RESULT3. They also show the filtering of individuals of ROUTE class through the reasoning process.
Table 1 Excerpts from the set of object properties

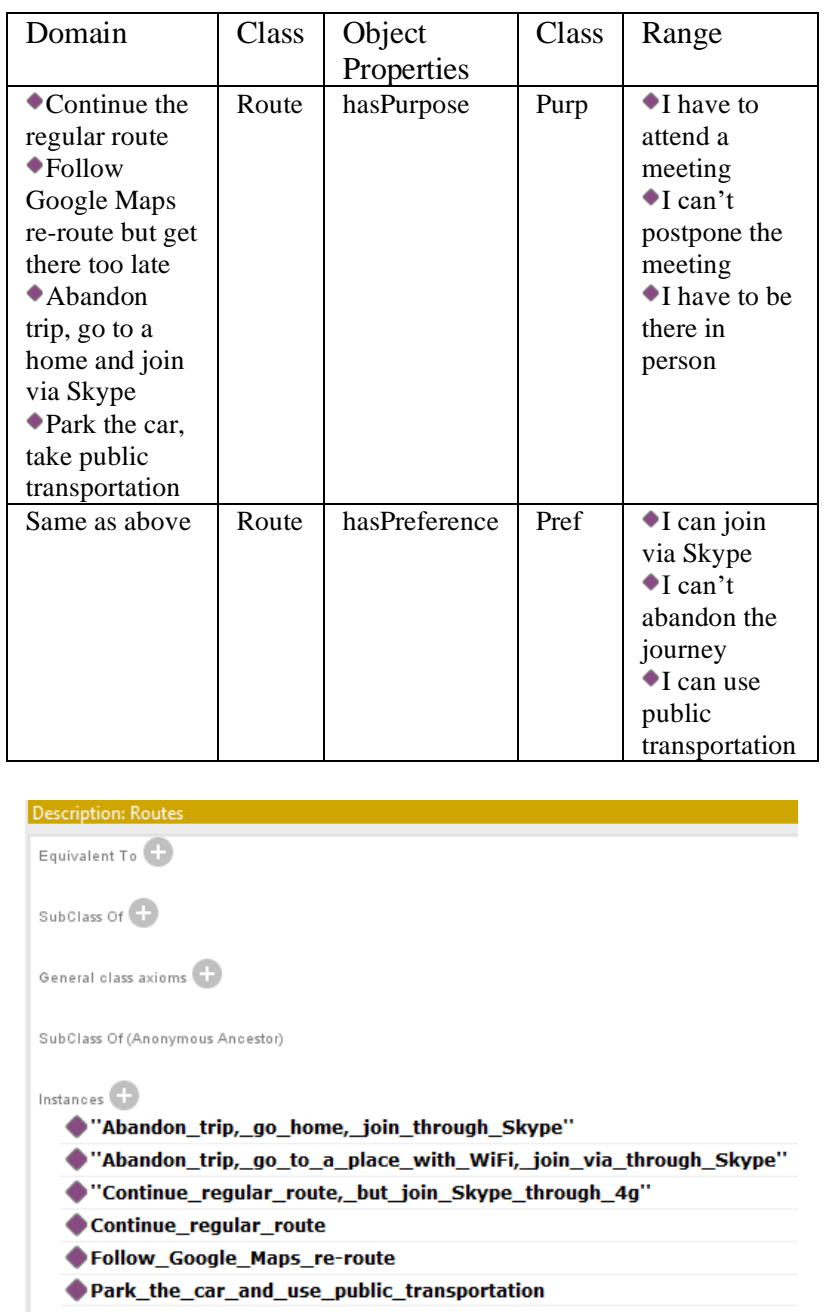

Figure 4: Individuals of ROUTE class

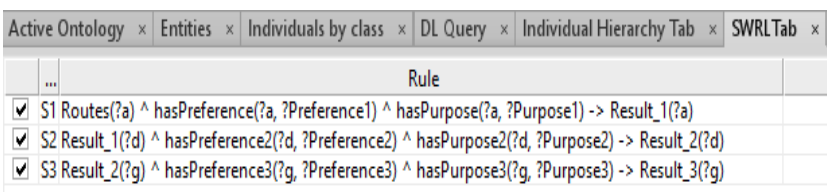

Figure 5: SWRL rules for reasoning upon the OWL model from Figure 3

Each of these screens are results of running SWRL rules S2 and S3. The final result of reasoning in Figure 8 says that the best option for Mr Lars is to: Park the car in the nearest car park and continue with public transport” 


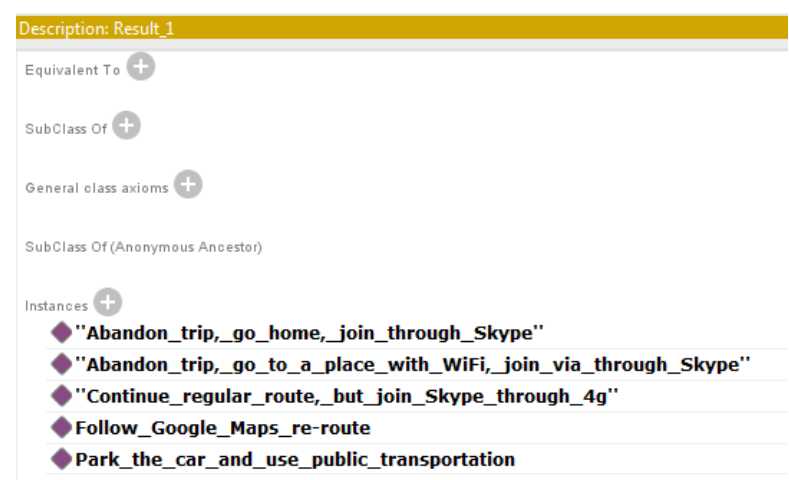

Figure 6: Individuals of ROUTE_1 class after running SWRL rule S1 (from Figure 4)

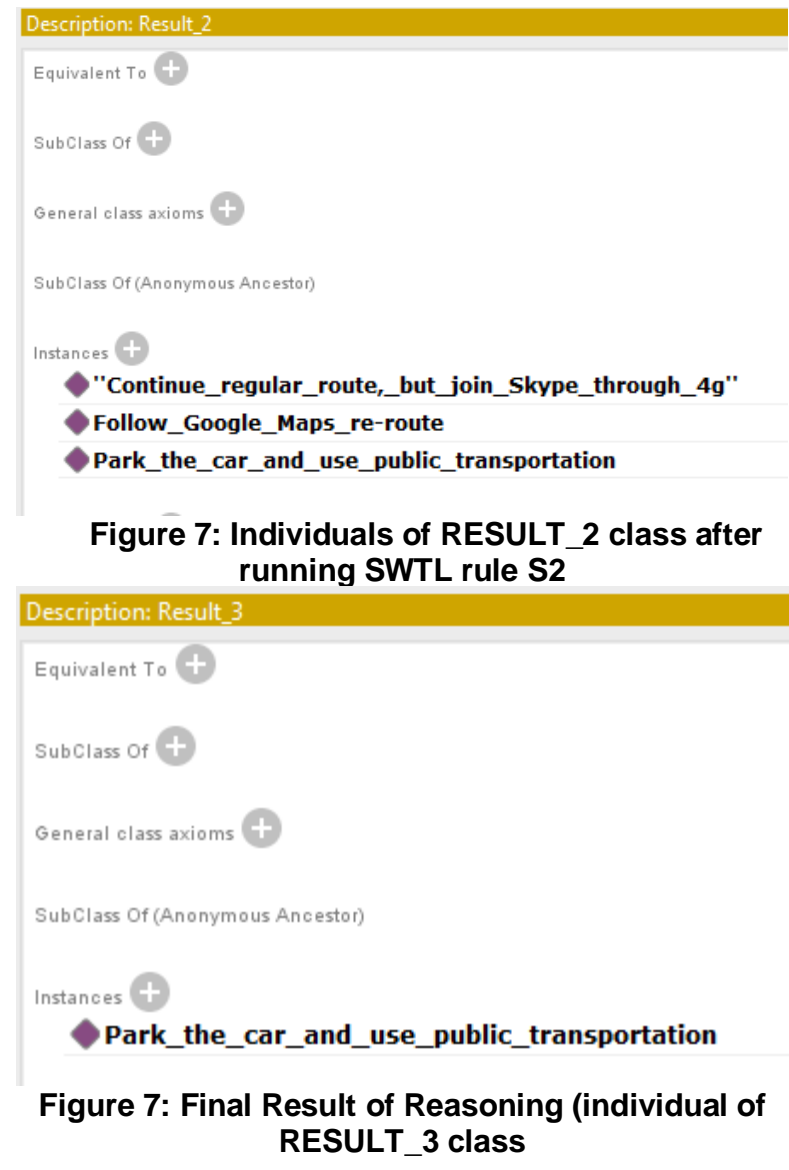

\section{Conclusions}

This paper promotes a new way of thinking for addressing congestion in urban traffic, by looking at possibilities of raising awareness that individual drivers do share their own responsibilities for creating traffic congestion. Any attempt to re-think every car journey, by looking at personal goals and preferences of drivers and giving a personalized advice on addressing traffic congestion, is beneficial. Our longterm interest in using SWT for addressing personalized decision making across many domains of interest [6571], proved to work very well in this particular domain. A prototype, as a software application generated from a generic SA, is reusable for two reasons:

a) The generic SA model has been defined, which enables reasoning and allows data sharing across interested parties in urban traffic.

b) The generic reasoning model, which is tested using the study of Oslo traffic municipality, works in any environment and in any city in the world.

Specificity of the implementation of the solution is solely in the individuals of the proposed ontologies and inferences, allowed though SWRL rules. Therefore computational models and SWRL rules in particular are generic and work everywhere. There is a very small step between this prototype and the full scale implementation of the final product. It has been already mentioned in section 5.1. that: (a) the SA from Figure 1 is feasible to implement in any IDE, including Android bundle, (b) SWRL rules are efficient computations which infer desirable result very fast, and (c) OWL-API makes reasoning with SWRL transparent to the user of the application.

These types of software applications do function smoothly, regardless which computational pathways from Figure 1 we wish to perform. The authors sincerely hope that this research would attract attention of traffic management institutions across urban world.

\section{References}

[1] Cambridge Dictionary. URBANIZATION | meaning in the Cambridge English Dictionary. 2019, https://dictionary.cambridge.org/dictionary/english/urbanizati on

[2] United Nations, $68 \%$ of the world population projected to live in urban areas by 2050, says UN. 2018., https://www.un.org/development/desa/en/news/population/ 2018-revision-of-world-urbanization-prospects.html

[3] K. Kourtit, P. Nijkamp, and H. Scholten, "The Future of the New Urban World". International Planning Studies, 2015. 20(1-2): p. 4-20.

[4] M. Chen et al., "The Global Pattern of Urbanization and Economic Growth: Evidence from the Last Three Decades". PLOS ONE, 2014. 9(8): p. 1-15.

[5] Wired. Visualising 1 billion cars. 2012 , https://www.wired.com/2012/07/1-billion-cars-infographic/

[6] International Organization of Motor Vehicle Manufacturers, Vehicles in use. 2019, http://www.oica.net/category/vehicles-in-use/

[7] International Organization of Motor Vehicle Manufacturers, 2018 Production Statistics. 2019, 
http://www.oica.net/category/production-statistics/2018statistics/

[8] Bureau of Transport Statistics, Number of U.S. Aircraft, Vehicles, Vessels, and Other Conveyances. 2018. https://www.bts.gov/content/number-us-aircraft-vehiclesvessels-and-other-conveyances

[9] U. S. Census Bureau for the United States, U.S. and World Population Clock. 2019, https://www.census.gov/popclock/

[10] A. Skabardonis., P. Varaiya, and K.F. Petty, "Measuring Recurrent and Nonrecurrent Traffic Congestion". Transportation Research Record, 2003. 1856(1): p. 118-124.

[11] Department for Transport, "Commuting trends in England $1988 \quad-\quad 2015 ”$. https://assets.publishing.service.gov.uk/government/uploads/ system/uploads/attachment data/file/657839/commuting-inengland-1988-2015.pdf

[12] T. van Vuren, and D. Leonard, "Urban congestion caused by incidents". Traffic Engineering and Control, 1994. 35: p. 422-429.

[13] A.M. de Souza et al., "Traffic management systems: A classification, review, challenges, and future perspectives". International Journal of Distributed Sensor Networks, 2017.

[14] A. Downs, The law of peak-hour expressway congestion. Traffic Quarterly, 1962. 16(3).

[15] C.N. Parkinson, The law and the profits. 1960: Houghton Mifflin.

[16] A. Downs, Still Stuck in Traffic : Coping with PeakHour Traffic Congestion. 2005: Brookings Institution Press.

[17] C. Li, S.G. Anavatti, and T. Ray, "Application of a noncooperative game theory based traffic assignment". in International Conference on Advances in Computing, Communications and Informatics (ICACCI), India, 2013

[18] D. Burns et al., "Acid Rain and its Environmental Effects: Recent Scientific Advances". Atmospheric Environment, 2016. 146: p. 1-4.

[19] J. Fenger, "Air pollution in the last 50 years - From local to global". Atmospheric Environment, 2009. 43(1): p. 13-22.

[20] H. Link et al., The Costs of Road Infrastructure and Congestion in Europe. Physica-Verlag GmbH \& Co, 1999.

[21] Inrix, INRIX 2018 Global Traffic Scorecard. 2019. http://inrix.com/

[22] G.-L. Chang and H. Xiang, "The relationship between congestion levels and accidents". Maryland State Highway Administration, 2003.

[23] C. Wang, "The relationship between traffic congestion and road accidents: an econometric approach using GIS" PhD Thesis. Loughborough University. 2010.
[24] S. A. C. S. Jayasooriya and Y. M. M. S. Bandara, "Measuring the Economic costs of traffic congestion," 2017 Moratuwa Engineering Research Conference (MERCon), Sri Lanka, 2017, pp. 141-146

[25] McKinsey \& Company, "Urban commercial transport and the future of mobility". 2017. https://www.mckinsey.com/business-functions/sustainability/ our-insights/urban-commercial-transport-and-the-future-ofmobility

[26] C. Pope et al., "Lung Cancer, Cardiopulmonary Mortality, and Long-Term Exposure to Fine Particulate Air Pollution". JAMA : the journal of the American Medical Association, 2002. 287: p. 1132-41.

[27] H. Khreis, K. de Hoogh, and M.J. Nieuwenhuijsen, "Full-chain health impact assessment of traffic-related air pollution and childhood asthma". Environment International, 2018. 114: p. 365-375.

[28] F. Mateen, and R. Brook, "Air Pollution as an Emerging Global Risk Factor for Stroke". JAMA : the journal of the American Medical Association, 2011. 305: p. 1240-1.

[29] J.S. Apte et al., “Ambient PM2.5 Reduces Global and Regional Life Expectancy". Environmental Science \& Technology Letters, 2018. 5(9): p. 546-551.

[30] B.G. Miller, Coal Energy Systems. 2004: Elsevier Science \& Technology. 103-103.

[31] M. Greenstone and C.Q. Fan, "Introducing the Air Quality Life Index - Twelve Facts about Particulate Air Pollution, Human Health, and Global Policy". Energy Policy Institute at the University of Chicago, 2018.

[32] C. Pope, M. Ezzati, and D. Dockery, "Fine-Particulate Air Pollution and Life Expectancy in the United States". The New England journal of medicine, 2009. 360: p. 376-86.

[33] M. Börjesson et al., "The Stockholm congestion charges -5 years on. Effects, acceptability and lessons learnt”. Transport Policy, 2012. 20: p. 1-12.

[34] C.P. Green, J.S. Heywood, and M. Navarro, "Traffic accidents and the London congestion charge". Journal of public economics, 2016. 133: p. 11-22.

[35] J. Coria et al., "Air pollution dynamics and the need for temporally differentiated road pricing". Transportation Research Part A: Policy and Practice, 2015. 75: p. 178-195.

[36] J. Coria, and X.-B. Zhang, "Optimal environmental road pricing and daily commuting patterns". Transportation Research Part B: Methodological, 2017. 105: p. 297-314.

[37] C. Johansson, L. Burman, and B. Forsberg, "The effects of congestions tax on air quality and health". Atmospheric Environment, 2009. 43(31): p. 4843-4854. 
[38] M. Gibson and M. Carnovale, "The effects of road pricing on driver behavior and air pollution". Journal of Urban Economics, 2015. 89: p. 62-73.

[39] J.W. Wedel, B. Schünemann, and I. Radusch. "V2Xbased traffic congestion recognition and avoidance". in 10th International Symposium on Pervasive Systems, Algorithms, and Networks, Taiwan 2009

[40] S. Dornbushand A. Joshi. StreetSmart traffic: "Discovering and disseminating automobile congestion using VANET's". in IEEE 65th Vehicular Technology ConferenceVTC, 2007 Spring.

[41] G.S. Khekare and A.V. Sakhare, "A smart city framework for intelligent traffic system using VANET" Proc. of International Mutli-Conference on Automation, Computing, Communication, Control and Compressed Sensing (iMac4s), India, March 2013

[42] G. Dimitrakopoulos, and P. Demestichas, "Systems Based on Cognitive Networking Principles and Management Functionality". IEEE Veh. Technol, 2010. 5: p. 77-84.

[43] S. Djahel et al., "A communications-oriented perspective on traffic management systems for smart cities: Challenges and innovative approaches". IEEE Communications Surveys \& Tutorials, 2015. 17(1): p. 125151.

[44] S. Sharma et al., "Traffic light priority control for emergency vehicle using RFID". Int. J. Innovative Eng. Technology, 2013. 2(2): p. 363-366.

[45] E. Walraven, M.T.J. Spaan, and B. Bakker, "Traffic flow optimization: A reinforcement learning approach". Engineering Applications of Artificial Intelligence, 2016. 52: p. 203-212.

[46] R.C. Carlson et al., "Optimal mainstream traffic flow control of large-scale motorway networks". Transportation Research Part C: Emerging Technologies, 2010. 18(2): p. 193-212.

[47] G. Horng, J. Li, and S. Cheng. "Traffic congestion reduce mechanism by adaptive road routing recommendation in smart city", Proc. of Inter. Conf. on Consumer Electronics, Communications and Networks., China, Nov 2013.

[48] P. Wang et al. "Detection of unwanted traffic congestion based on existing surveillance system using in freeway via a CNN-architecture trafficnet". Proc. Of 13th IEEE Conf. on Industrial Electronics and Applic. (ICIEA), China, May 2018 [49] D. Ma et al., "Estimating Maximum Queue Length for Traffic Lane Groups Using Travel Times from VideoImaging Data". IEEE Intelligent Transportation Systems Magazine, 2018. 10(3): p. 123-134.

[50] M. Yang, R. Jhang, and J. Hou, "Traffic flow estimation and vehicle-type classification using vision-based spatialtemporal profile analysis". IET Computer Vision, 2013. 7(5): p. 394-404.

[51] X. Kong et al., "Urban traffic congestion estimation and prediction based on floating car trajectory data". Future Generation Computer Systems, 2016. 61: p. 97-107.

[52] H. Park and A. Haghani, "Optimal number and location of Bluetooth sensors considering stochastic travel time prediction". Transportation Research Part C: Emerging Technologies, 2015. 55: p. 203-216.

[53] H. Dezani et al., "Optimizing urban traffic flow using Genetic Algorithm with Petri net analysis as fitness function". Neurocomputing, 2014. 124: p. 162-167.
[54] S. Çolak, A. Lima, and M.C. Gonzalez, "Understanding congested travel in urban areas". Nature communications, 2016. 7: p. 10793.

[55] G. Gutierrez et al. "Agent-based framework for Advanced Driver Assistance Systems in urban environments", Proceedings of 17th International Conf. on Information Fusion (FUSION) Salamanca Spain,July 2014

[56] A. Armand, D. Filliat, and J. Ibañez-Guzman. "Ontology-based context awareness for driving assistance systems". in Proceedings of IEEE Intelligent Vehicles Symposium, MI, USA, June 2014.

[57] M. Hülsen, J.M. Zöllner, and C. Weiss. "Traffic intersection situation description ontology for advanced driver assistance". in Proc. of IEEE Intelligent Vehicles Symposium (IV). Baden-Baden, Germany, June 2011

[58] M. Houda et al. "A public transportation ontology to support user travel planning". in Proc. $4^{\text {th }}$ Intern. Conf. on Research Challenges in Information Science (RCIS). France, May, 2010.

[59] A.S. Niarakiand and K. Kim, "Ontology based personalized route planning system using a multi-criteria decision making approach". Expert Systems with Applications, 2009. 36(2): p. 2250-2259.

[60] S. Fernandez, and T. Ito. "Driver Behavior Model Based on Ontology for Intelligent Transportation Systems". in Proc. IEEE 8th Intern. Conference on Service-Oriented Computing and Applications (SOCA). Italy, October 2015.

[61] Y. Akagi, "Ontology based collection and analysis of traffic event data for developing intelligent vehicles". Proc. of $6^{\text {th }}$ IEEE Global Conference on Consumer Electronics (GCCE), Japan, Oct. 2017.

[62] M. Buechel et al. "Ontology-based traffic scene modeling, traffic regulations dependent situational awareness and decision-making for automated vehicles". in Proc. $28^{\text {th }}$ IEEE Intelligent Vehicles Symp. (IV).Ca, US, June 2018.

[63] M. Haggag and D. R. Mahmoud, "OnTraJaCS: Ontology based Traffic Jam Control System". International Journal of Computer Applications, 2012. 60: p. 6-16.

[64] S. Balakrishna and M. Thirumaran, "Semantic Interoperable Traffic Management Framework for IoT Smart City Applications". EAI Endorsed Transactions on Internet of Things, 2018. 4: p. 185-211.

[65] R. Juric, K. Moholth McClenaghan,'Towards Semantic Classification of Constituent Parts of the Internet of Vehicles", Proc. of $24^{\text {th }}$ SDPS 2019 Conf. Taiwan, July 2019. [66] R. Juric, "Semantic model for Creating an Instance of IoT", Proceedings of $4^{\text {th }}$ IEEE CyberScieTech Conference, Japan, August 2019.

[67] M. Tarabi, R.Juric, "Software Architectures for Smart Applications in the Management of Chronic Diseases: A Case Study of Reversibility of Diabetes 2", Proceedings of $51^{\text {st }}$ HICSS Conference, HI, US, January 2018.

[68] R. Juric, "Could Semantic Web Technologies Create New Computational Models outside Semantic Web", Proceedings of $21^{\text {st }}$ SDPS 2016 Conf. Fl, US. Dec. 2016 [69] A. Shakil, R. Juric, Addressing Urban Traffic Congestion Using Individual Driver's Preferences and Reasoning upon the Purpose and Goals of Car Journeys, Proceedings of SDPS 2018 Workshop on Accountability of AI, Italy, Dec. 2018. 